\title{
THE EMISSION SPECTRA OF RADIOWEAK QUASARS.
}

\section{THE FIR EMISSION}

\author{
MARTINA NIEMEYER and PETER L. BIERMANN \\ Max-Planck-Institut für Radioastronomie \\ Auf dem Hügel 69 \\ D-53121 Bonn, Germany
}

The far-infrared (FIR) spectra of radioweak quasars show a steep rise from the $\mathrm{mm}$ to the FIR wavelengths with an spectral index often $\alpha \geq 2.5$, and a slow decrease beyond the FIR with $\alpha \simeq-1$. A comparison of the FIR luminosity with both radio and X-ray emission demonstrates, that only the active nuclei can provide the energy to heat the dust. We propose that the heating is due to the central engine via relativistic particles. These particles originate from a putative source on the symmetry axis close to the central engine and diffuse through a tenuous galactic halo to heat dusty molecular clouds in a disk configuration. We demonstrate that this mechanism can produce the FIR spectra with reasonable assumptions, and compare them with observations (Chini et al. 1989). We examine the diffusion through the halo and the energy deposition in the disk for two types of source (pointsource, line source, intensity decrease with $z^{-\beta}$ ) and a diffusion coefficient, dependent on the spherical radius $r\left(D \propto r^{\gamma}\right)$. We obtain the total energy deposition in the disk as a function of the disk radius. For the calculation of the FIR spectra, one must determine the dust temperature. We construct a heat balance that takes into account the absorbed and emitted emission of energy. The calculated dust temperature distribution depends on two parameter: the radial dependence of the diffusion coefficient and the z-dependence of the line source. Then we calculate the FIR spectra of a disk in the wavelength range 10 to 1300 $\mu \mathrm{m}$. We determine the luminosity of a finite disk, where the limits are determined by the maximal and minimal temperature $\left(T_{D, \max }=1500 \mathrm{~K}\right.$ given by the dust destruction, $T_{D, \min }=20 \mathrm{~K}$ given by a transition to dust heating from young stars in the extended disk).

We find that for a diffusion coefficient in the region above the disk, which scales linearly with the spherical radius $r$, a line source, which intensity decreases by $z^{-1}$ and a total source luminosity of 3 to 10 times the observed infrared luminosity, we can reproduce and interpret the spectra of radioweak quasars in the $\mathrm{mm}$ to the near infrared range.

\section{References}

Chini R., Kreysa E., Biermann P.L.: 1989, Astronomy and Astrophysics 219, pp. 87-97

Niemeyer M., Biermann P.L.: 1993, Astronomy and Astrophysics in press

350

T. J.-L. Courvoisier and A. Blecha: Multi-Wavelength Continuum Emission of AGN, 350.

(C) 1994 IAU. Printed in the Netherlands. 